\title{
Regulation of the innate IL-17 response to Candia albicans in the oral mucosa
}

Florian Sparber ${ }^{1}$, Tamas Dolowschiak², Sarah Mertens ${ }^{1}$, Laura Lauener ${ }^{1}$, Björn E. Clausen ${ }^{3}$, Nicole Joller², Patrizia Stoitzner ${ }^{4}$, Roxane Tussiwand ${ }^{5}$, Salomé Leibundgut-Landmann ${ }^{1}$

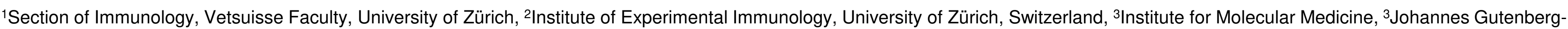
University, Mainz, Germany, ${ }^{4}$ Department of Dermatology, Medical University Innsbruck, Austria, ${ }^{5}$ Department of Biomedicine, University of Basel, Switzerland

University of

Zurich $^{\mathrm{uzH}}$

Contact: florian.sparber@uzh.ch and salome.leibundgut-landmann@uzh.ch

1. Introduction \& Aim

Interleukin-17 (IL-17) plays a key role in protective immunity against $C$. albicans, as demonstrated by increased susceptibility to superficial $C$. albicans infections in patients with genetic defects in the IL-17 pathway and in experimentally infected IL-17-deficient mice. Yet, the cellular source of IL-17 and its regulation during acute infection remains a matter of debate. Using the mouse model of oropharyngeal candidiasis (OPC), we visualized IL-17 protein production at a single cell level to determine

$X$ the identity of innate IL-17 producing cells in the oral cavity

$X$ the regulatory mechanisms that instruct IL-17 production by these cells at the onset of infection

1. IL-17A is expressed by a tripartite population of CD90+ leukocytes in the infected tongue.

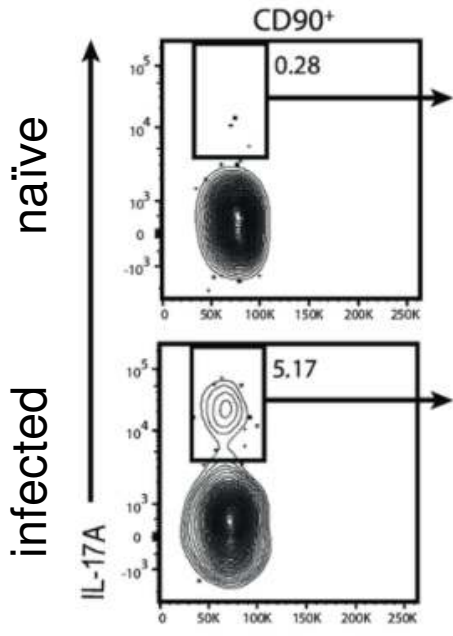

FSC-A $\longrightarrow$

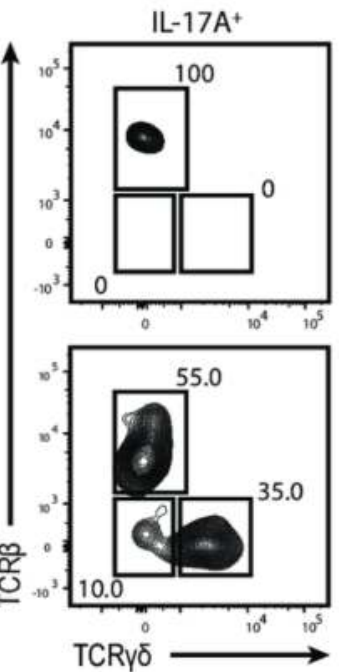

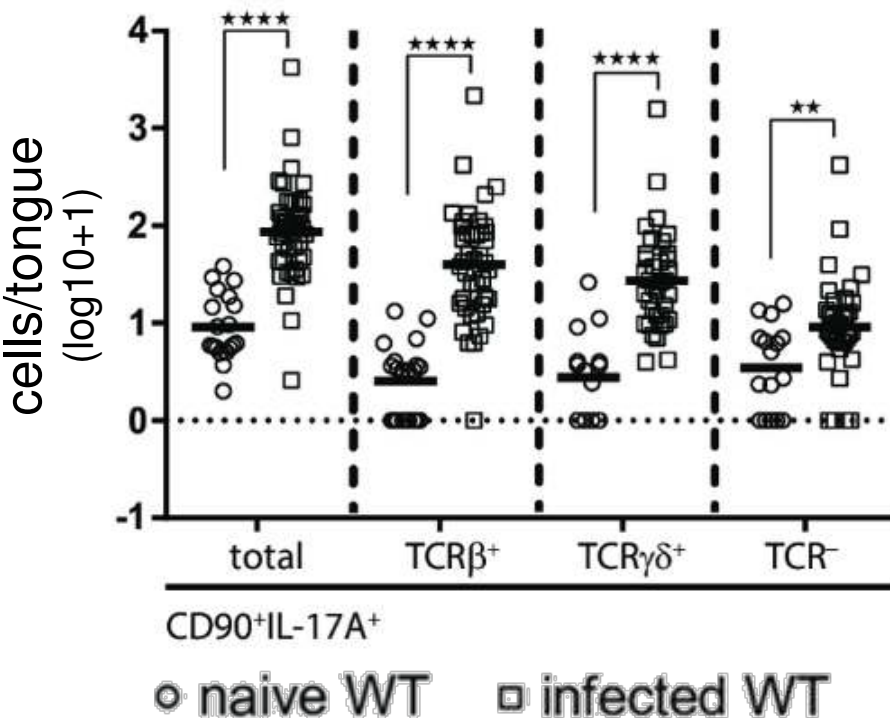

$\circ$ naive WT $\square$ infected WT
Intracellular cytokine staining and flow cytometric analysis of IL-17A protein expression in tongue leukocytes on day 1 p.i. demonstrates production of this key cytokine by three distinct CD90+ leukocyte populations comprising $\alpha \beta^{+}$T cells, $\gamma \delta+T$ cells and TCR- ILCs

3. Tissue-resident Langerin+ DCs produce all IL-17instructing factors during OPC: IL-1 $\beta$, IL-6 and IL-23.
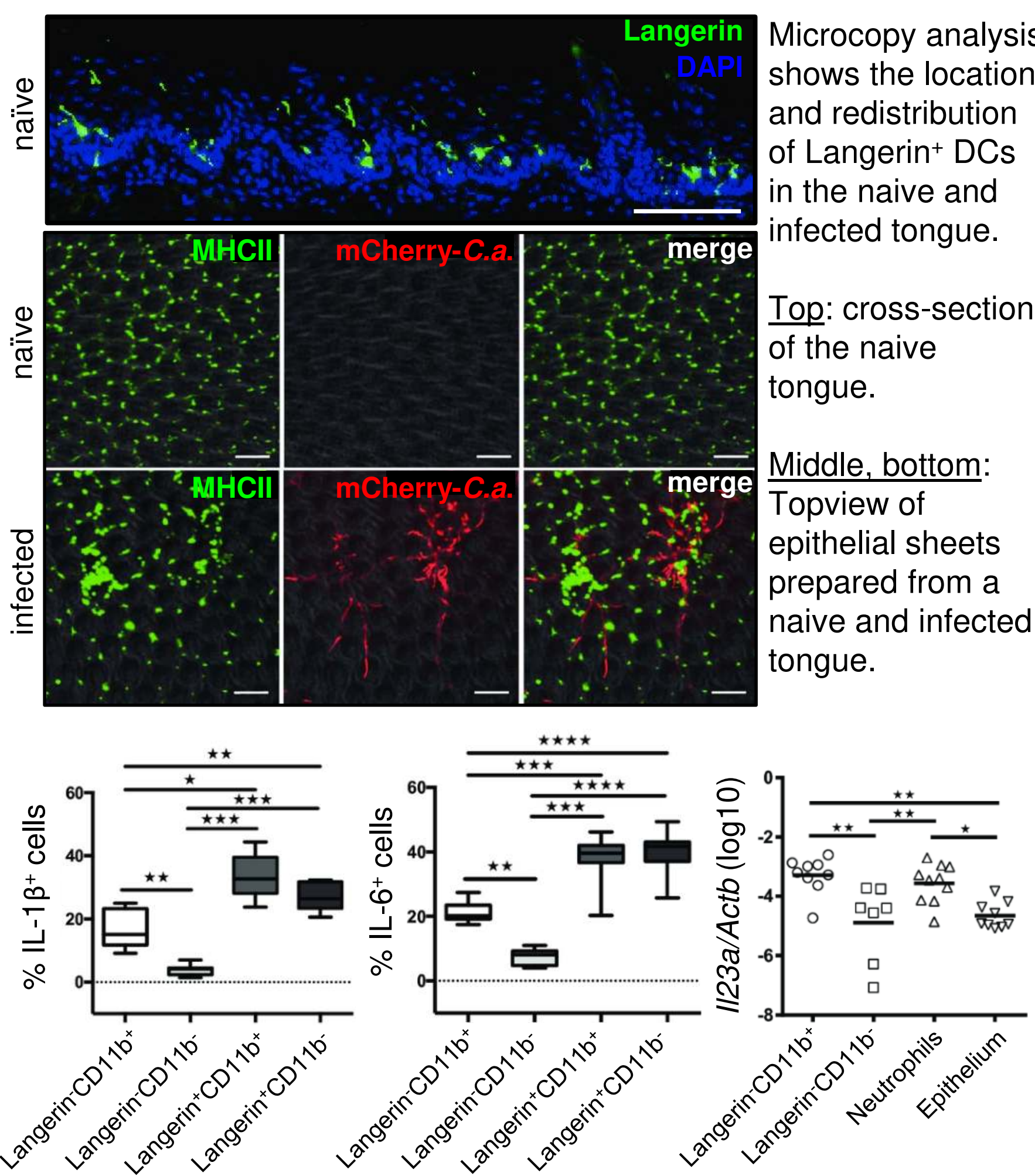

IL-1 $\beta$ and IL-6 production was quantified within the indicated tongue MNP/DC subsets by intracellular staining and flow cytometry. IL-23 mRNA was quantified by qRT-PCR in sorted cell subsets. Analysis on day 1 p.i.
2. IL-17A production during OPC relies on IL-23, IL-6 and IL-1 signaling in a partially redundant manner.
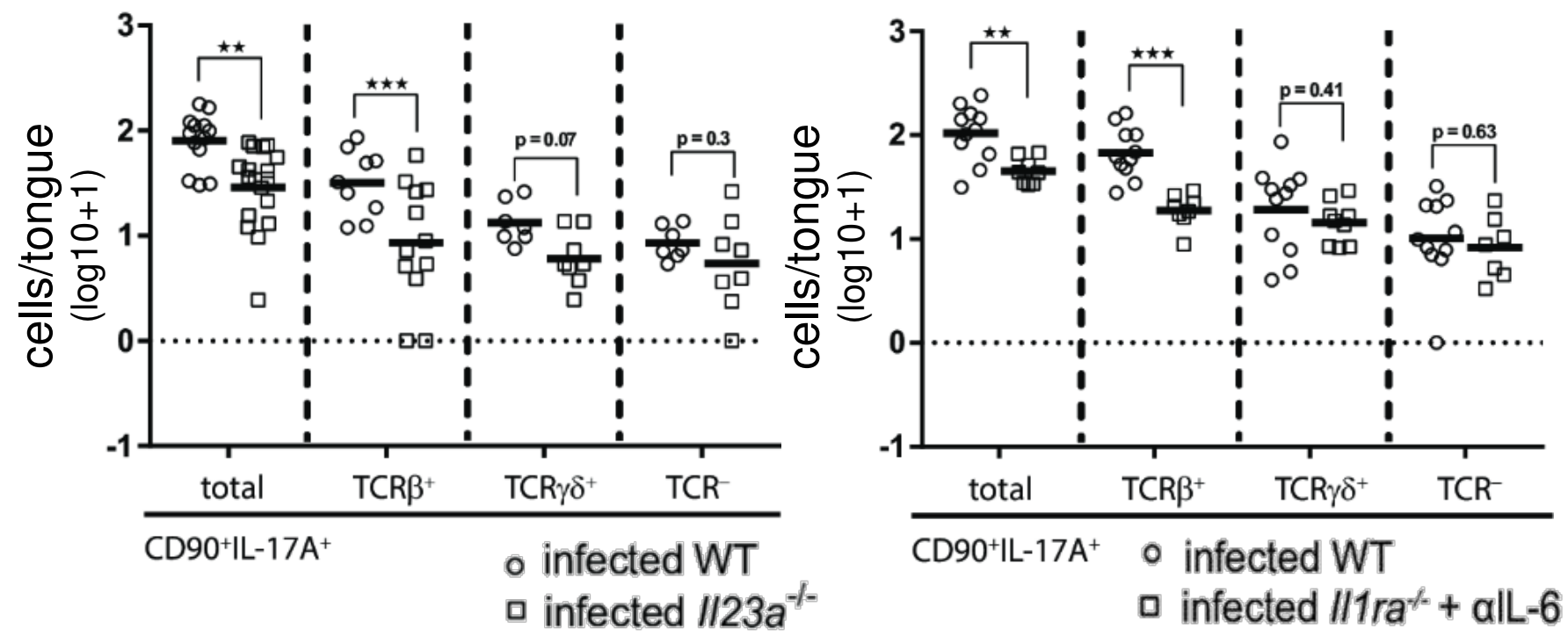

Mice deficient in IL-23 (left) or IL-1/IL-6 signaling (right) show reduced numbers of CD90+IL-17A+ lymphocytes on day 1 post-infection compared to WT controls. Tongue leukocytes were analyzed by intracellular cytokine staining and flow cytometry as in Fig. 1.

4. In vivo depletion of Langerin+ DCs impairs IL-17A production by CD90+ leukocytes during OPC.
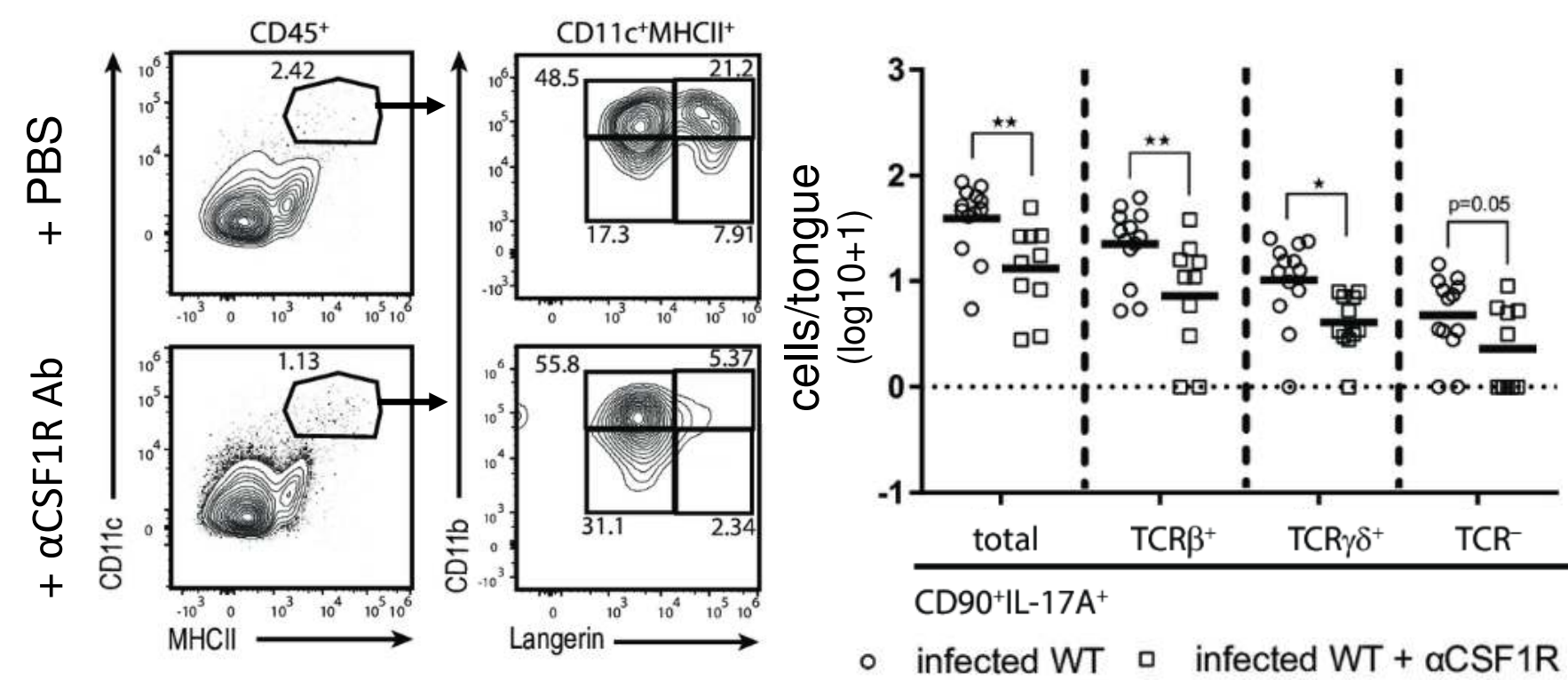

Antibody-mediated blocking of CSF1R signaling in infected WT mice results in depletion of Langerin ${ }^{+} \mathrm{DCs}$ and consequently in a reduction of IL-17A production by all three CD90+ cell subsets (see Figure 1) on day 1 p.i.

5. Concept: Langerin+ DCs coordinate the innate IL-17 response to oral Candida infection.

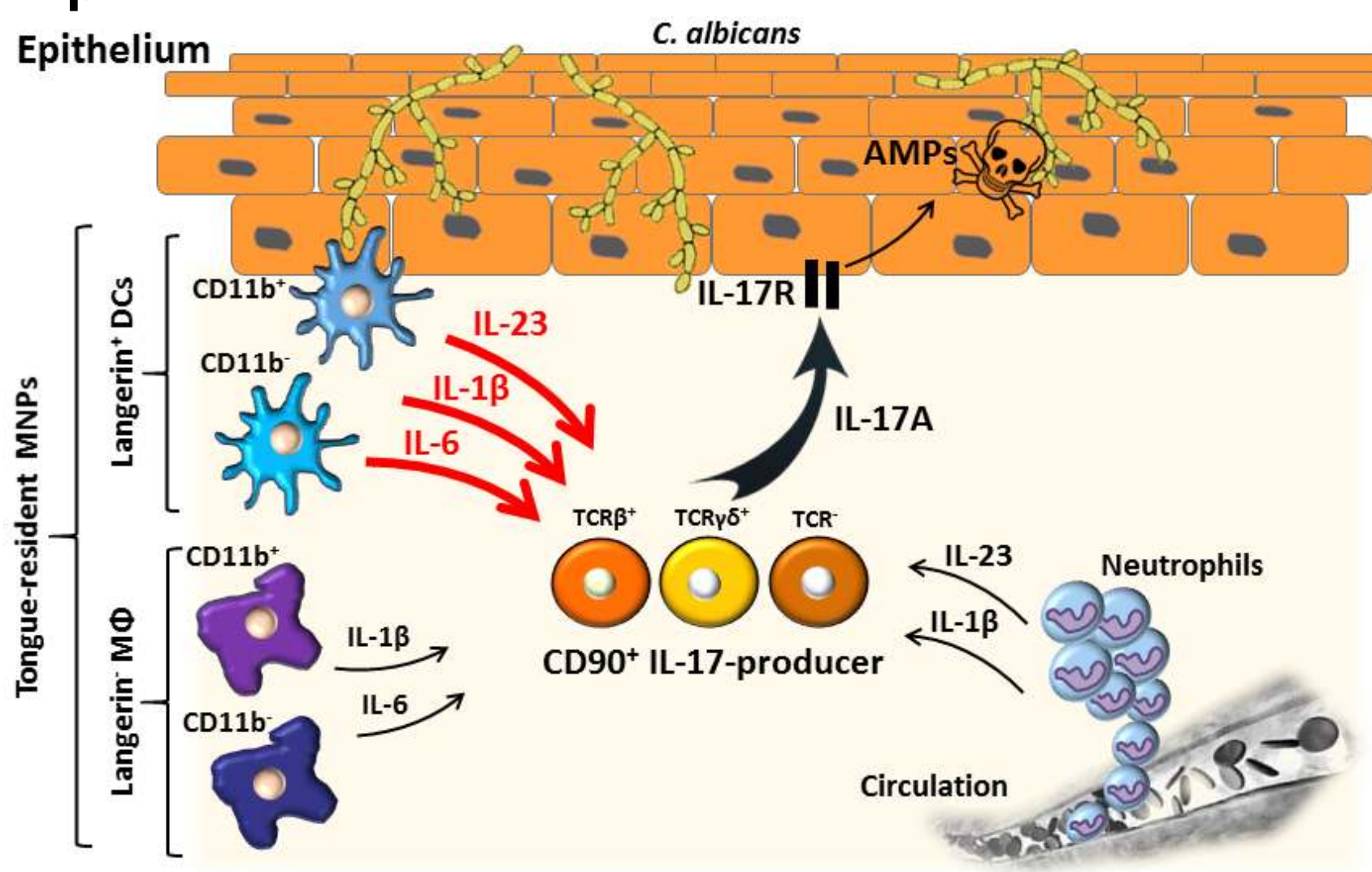

MNPs: mononuclear phagocytes, DCs: dendritic cells, IL-17R: IL-17 receptor; AMP: antimicrobial peptides 